\title{
ISEE_Wave: interactive plasma wave analysis tool
}

\author{
Shoya Matsuda ${ }^{* *} \mathbb{D}$, Yoshizumi Miyoshi², Satoko Nakamura², Masahiro Kitahara², Masafumi Shoji², \\ Tomoaki Hori ${ }^{2}$, Shun Imajo ${ }^{2}$, Chae-Woo Jun², Satoshi Kurita ${ }^{3}$, Yoshiya Kasahara ${ }^{4}$, Ayako Matsuoka ${ }^{5}$ and \\ Iku Shinohara'
}

\begin{abstract}
We have developed ISEE_Wave (Institute for Space-Earth Environmental Research, Nagoya University - Plasma Wave Analysis Tool), an interactive plasma wave analysis tool for electric and magnetic field waveforms observed by the plasma wave experiment aboard the Arase satellite. ISEE_Wave provides an integrated wave analysis environment on a graphical user interface, where users can visualize advanced wave properties, such as the electric and magnetic field wave power spectra, wave normal polar angle, polarization ellipse, planarity of polarization, and Poynting vector angle. Users can simply select a time interval for their analysis, and ISEE_Wave automatically downloads the waveform data, ambient magnetic field data, and spacecraft attitude data from the data archive repository of the ERG Science Center, and then performs necessary coordinate transformation and spectral matrix calculation. The singular value decomposition technique is used as the core technique for the wave property analysis of ISEE_Wave. On-demand analysis is possible by specifying the parameters of the wave property analysis as well as the plot styles using the graphical user interface of ISEE_Wave. The results can be saved as image files of plots and/or a tplot save file. ISEE_Wave aids in the identification of fine structures of observed plasma waves, wave mode identification, and wave propagation analysis. These properties can be used to understand plasma wave generation, propagation, and waveparticle interaction in the inner magnetosphere. ISEE_Wave can also be applied to general waveform data observed by other spacecraft by using the plug-in procedures to load the data.
\end{abstract}

Keywords: Plasma waves, Direction finding, Arase satellite, SPEDAS

\section{Introduction}

Plasma waves in the magnetosphere play an important role in the acceleration and dissipation of charged particles. Cross-energy coupling in the terrestrial inner magnetosphere is mainly supported by the existence of several types of plasma waves. For example, energetic electron acceleration and precipitation loss are driven by whistler-mode chorus waves in the inner magnetosphere (Kasahara et al. 2018; Ozaki et al. 2019; Foster

*Correspondence: matsuda@stp.isas.jaxa.jp

1 Institute of Space and Astronautical Science, Yoshinodai, Sagamihara 252-5210, Japan

Full list of author information is available at the end of the article et al. 2017; Reeves et al. 2013). Wave polarization, which is an important plasma wave parameter, characterizes the wave mode according to the dispersion relation of plasma waves. The wave normal angle is also an important feature of plasma waves and it characterizes wave propagation. Colpitts et al. (2020) reported a simultaneous measurement of whistler-mode chorus waves along a field line obtained by Arase and Van Allen probes. They found that the wave normal angle of the waves is more oblique in the off-equatorial region than in the equatorial region and concluded that the result is consistent with the theory of plasma wave propagation and ray-tracing results. Similar multipoint measurements of very low frequency/extremely low frequency (VLF/ELF) plasma waves were reported by Martinez-Calderon et al. (2020). 
The aforementioned wave parameters are also important for evaluating theoretical properties of wave-particle interactions, such as the pitch-angle diffusion coefficient due to whistler-mode chorus waves (Horne et al. 2005).

Recently, three-dimensional VLF/ELF magnetic field measurement has been carried out by most of spacecraft. Two-dimensional (spin plane) or three-dimensional (spin plane and spin axis) electric field measurement is also performed by spinning spacecraft, such as the Van Allen probes (Mauk et al. 2013), Magnetospheric Multiscale (MMS) spacecraft (Burch et al. 2016), Time History of Events and Macroscale Interactions during Substorms (THEMIS) spacecraft (Angelopoulos 2008), and Exploration of energization and Radiation in Geospace (ERG/ Arase) satellite (Miyoshi et al. 2018a). The wave normal angles and polarization of plasma waves can be derived from these multi-component measurements, although the method is more complicated than the power spectrum calculation (obtained by determining the absolute value of the Fourier transformed complex spectrum). Thus, the evaluation of such wave properties can be a barrier for non-experts in plasma wave analysis.

Propagation analysis of STAFF-SA (spatio-temporal analysis of field fluctuations, spectrum analyzer) data with coherency tests (PRASSADCO) is a character user interface (CUI) tool for analyzing plasma wave data of multi-component measurements (http://aurora. troja.mff.cuni.cz/ santolik/PRASSADCO/). This tool provides an environment of wave propagation property analysis from spectral matrices observed by wave instruments such as Cluster/STAFF (Cornilleau-Wehrlin et al. 1997), Cassini/Radio and Plasma Wave Science (RPWS) (Gurnett et al. 2004), Polar/Plasma Wave Investigation (PWI) (Gurnett et al. 1995), and Van Allen probes/Electric and Magnetic Field Instrument Suite and Integrated Science (EMFISIS) (Kletzing et al. 2013). PRASSADCO enables users to obtain results of wave property analysis as publication-quality figures, and has been used in a number of papers (e.g., Santolík et al. (2001); Němec et al. (2005); Cornilleau-Wehrlin et al. (2003)). The Interface for a sPECtral Matrix ANalysis (iPECMAN, https://ipecman.ufa.cas.cz) was also developed as a web-based analysis tool based on PRASSADCO to analyze spectral matrix data observed by the STAFF-SA instrument aboard Cluster.

In this paper, we present an interactive plasma wave property analysis tool called ISEE_Wave (Institute for Space-Earth Environmental Research, Nagoya University - Plasma Wave Analysis Tool), which has recently been available for researchers being interested in plasma wave analysis. ISEE_Wave is a graphical user interface (GUI) tool implemented as a plug-in for Space Physics Environment Data Analysis System (SPEDAS; see Angelopoulos et al. (2019)). Unlike the previous tools using premade spectral matrix data, ISEE_Wave directly processes waveform data and thereby allows the user to construct spectral matrix data on-the-fly with a Fourier window, window width, and overlap length of the user's own. Because all functions implemented in ISEE_Wave can be used through the GUI, users can easily obtain publication-quality plots without using complicated commands. ISEE_Wave is written in interactive data language (IDL) and accordingly can be used on any platform and operating system where IDL is available. We present examples of wave property analysis using waveform data obtained by Arase. ISEE_Wave can be applied to waveform data observed by other spacecraft, such as the Van Allen probes, THEMIS spacecraft, MMS spacecraft by replacing the data load routines.

\section{Method}

ISEE_Wave provides an end-to-end analysis environment for plasma wave data. All functions of ISEE_Wave are provided in an integrated GUI program in IDL/SPEDAS. Without knowledge of IDL/SPEDAS commands, users can analyze waveform data obtained through the waveform capture/onboard frequency analyzer (WFC/OFA) (Matsuda et al. 2018), which is a sub-component of plasma wave experiment (PWE) (Kasahara et al. 2018a) aboard Arase. Figure 1 illustrates an overview of how ISEE_Wave processes plasma wave and related ancillary data on a user's computer. ISEE_Wave is a plug-in tool for IDL/SPEDAS and works in combination with the ERG plug-in provided by the ERG Science Center (Miyoshi et al. 2018c). ISEE Wave uses six types of Level-2 data products of Arase provided by the ERG Science Center: spacecraft orbit data (Miyoshi et al. 2018b), PWE/OFA-SPEC data (Kasahara et al. 2018b), PWE/WFC ancillary data, PWE/WFC waveform data in the Spinning satellite Geometry Axis (SGA) coordinates (Kasahara et al. 2020a, b), spacecraft attitude data, and spin-averaged ambient magnetic field data in the Despun Sun sector Inertia (DSI) coordinates obtained by the magnetic field experiment (MGF) (Matsuoka et al. 2018a, b). The first three products are used to visualize an overview of the plasma wave power spectra and the history of waveform observations, while the last three products are used to calculate the detailed wave properties. Because we use waveform data in the SGA coordinate system, we transform them to the DSI coordinate system using spacecraft attitude data. To obtain the wave normal angle, wave polarization, and Poynting vector angle with respect to the ambient geomagnetic field lines, we further transform the data to the geomagnetic field (MAG) coordinate system using DC magnetic field data measured by MGF. All of those data are automatically downloaded from the ERG Science Center. 
The PWE aboard Arase measures three magnetic field components and two electric field components in the inner magnetosphere (Kasahara et al. 2018a). The OFA measures electric and magnetic field wave power spectra and $2 \times 2$ electric and $3 \times 3$ magnetic field spectral matrices for the frequency range of several tens of $\mathrm{Hz}$ to $20 \mathrm{kHz}$ (Matsuda et al. 2018). Their spectra are continuously measured and we can thus monitor plasma wave activity and wave properties (e.g., polarization, wave normal angle, and Poynting vector angle) using data obtained by OFA. However, OFA data are not suitable for analyzing the wave properties of short duration events, such as discrete chorus elements, because the measurement cadence is much longer, 1 and $8 \mathrm{~s}$ for wave power spectra and spectral matrices, respectively. In contrast, WFC measures waveforms of two electric and three magnetic field components with a sampling frequency of $65.536 \mathrm{kHz}$. As reported by Matsuda et al. (2018), three modes are available in WFC: the chorus burst mode, the electromagnetic ion cyclotron (EMIC) burst mode, and the software-type wave-particle interaction analyzer (SWPIA) mode. The chorus burst mode measures two electric and three magnetic field waveforms with a sampling frequency of $65.536 \mathrm{kHz}$. The chorus burst mode intermittently observes continuous waveforms of a duration of 8-200 s, and the length of the continuous waveforms can be changed through an onboard command. During the rest period, the observed waveforms are compressed by the subband coding algorithm using the quadrature mirror filter (QMF) tree filter bank. This mode is designed for the measurement of whistler-mode plasma waves (whistler-mode chorus, plasmaspheric hiss, and electron cyclotron harmonic waves), while the EMIC burst mode is designed for measurement of EMIC and magnetosonic waves. The EMIC burst mode measures downsampled (1024 samples/s) waveforms using an onboard cascaded decimation filter. The SWPIA mode is a special mode to obtain continuous $65.536 \mathrm{kHz}$ sampling waveforms without onboard data compression. The quality of data obtained by the SWPIA mode is essentially the same as that of data obtained by the chorus burst mode. Note that these three modes are exclusively operated.

ISEE_Wave is designed for analyzing the data of these three modes. In Sections "Functions of ISEE Wave" and "Scientific applications", we present an analysis of 65.536 $\mathrm{kHz}$ sampling waveforms obtained by the chorus burst mode, as the waveform data observed by the EMIC burst mode are not currently publicly available. We can analyze the wave power spectra, polarization, and wave normal angle with a high time and frequency resolutions by calculating magnetic spectral matrices from the observed waveforms. Note that if we use only magnetic field spectral matrices, the calculated wave normal angle has an ambiguity in the sign of polarity because the correlation between electric and magnetic fields is not calculated. We can unambiguously determine the direction of the wave propagation vector ( $k$-vector) by calculating the Poynting vector

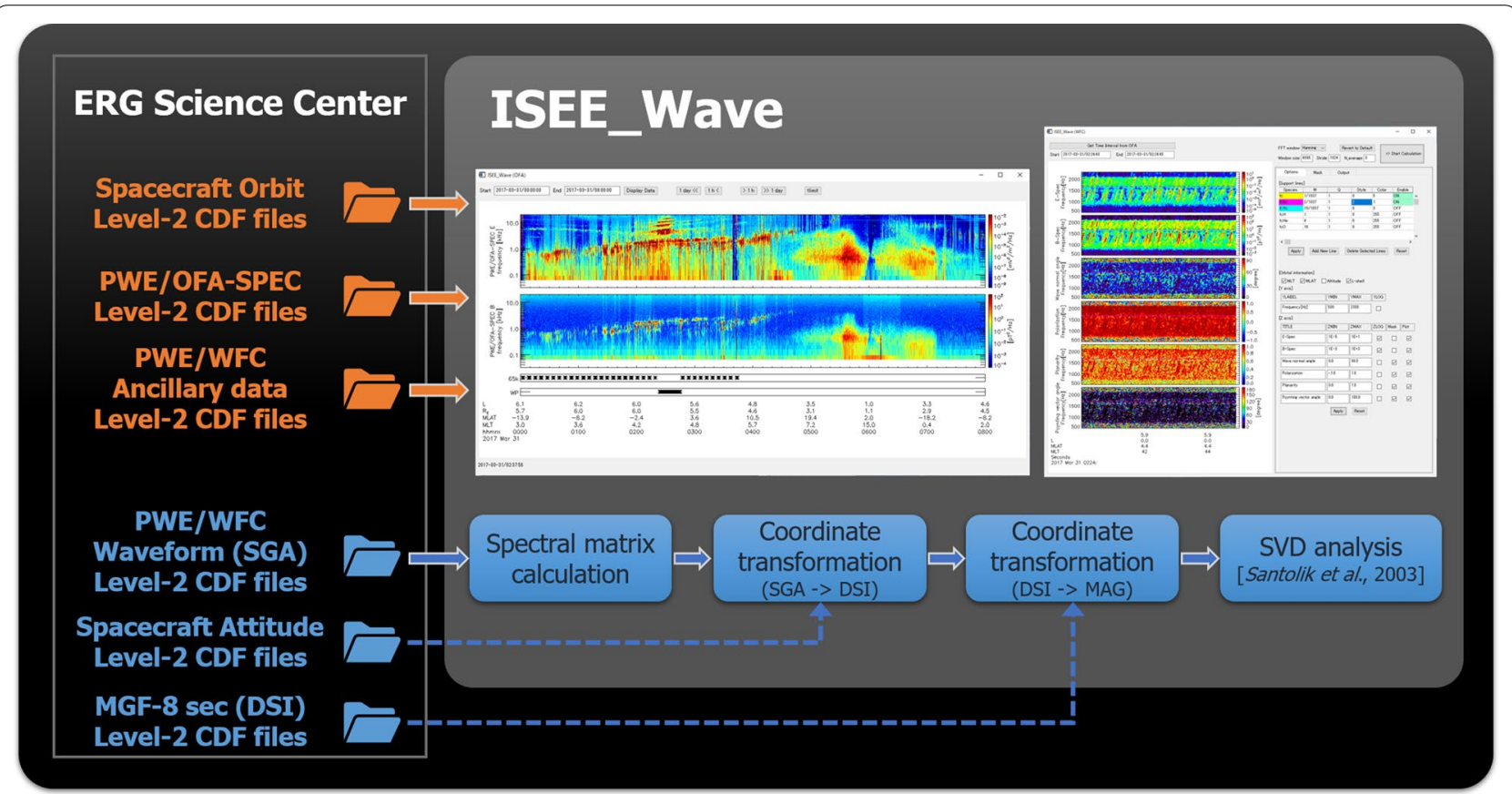

Fig. 1 Overview of the data-flow and internal processings of ISEE_Wave 
angle using both the electric and magnetic field complex spectra.

The singular value decomposition (SVD) technique (Santolík et al. 2003) is implemented in ISEE_Wave. ISEE_Wave calculates magnetic field spectral matrices $A$ by performing short-time Fourier transforms (STFT) of the waveforms observed by PWE/WFC: and magnetic field is not calculated. To determine the polarity of $k$-vector, we calculate the Poynting vector angle using electric and magnetic field complex spectra. The Poynting vector angle $\theta_{\mathrm{P}}$ can be derived as $\theta_{\mathrm{P}}=\arccos \left(S_{3} / \sqrt{S_{1}{ }^{2}+S_{2}{ }^{2}+S_{3}{ }^{2}}\right) . \quad \theta_{\mathrm{P}}=0^{\circ}$ and $180^{\circ}$ indicate parallel and anti-parallel propagation along the field line, respectively. $S_{1}, S_{2}$, and $S_{3}$ denote the three

$$
A=\left(\begin{array}{lll}
a_{00} & a_{01} & a_{02} \\
a_{10} & a_{11} & a_{12} \\
a_{20} & a_{21} & a_{22} \\
a_{30} & a_{31} & a_{32} \\
a_{40} & a_{41} & a_{42} \\
a_{50} & a_{51} & a_{52}
\end{array}\right)=\sum_{n=1}^{N}\left(\begin{array}{ccc}
\operatorname{Re}\left(\left|\mathrm{B}_{\mathrm{x}}^{(\mathrm{n})}\right|^{2}\right) & \operatorname{Re}\left(\mathrm{B}_{\mathrm{x}}^{(\mathrm{n})} \mathrm{B}_{\mathrm{y}}^{(\mathrm{n}) *}\right) & \operatorname{Re}\left(\mathrm{B}_{\mathrm{x}}^{(\mathrm{n})} \mathrm{B}_{\mathrm{z}}^{(\mathrm{n}) *}\right) \\
\operatorname{Re}\left(\mathrm{B}_{\mathrm{x}}^{(\mathrm{n})} \mathrm{B}_{\mathrm{y}}^{(\mathrm{n}) *}\right) & \operatorname{Re}\left(\left|\mathrm{B}_{\mathrm{y}}^{(\mathrm{n})}\right|^{2}\right) & \operatorname{Re}\left(\mathrm{B}_{\mathrm{y}}^{(\mathrm{n})} \mathrm{B}_{\mathrm{z}}^{(\mathrm{n}) *}\right) \\
\operatorname{Re}\left(\mathrm{B}_{\mathrm{x}}^{(\mathrm{n})} \mathrm{B}_{\mathrm{z}}^{(\mathrm{n}) *}\right) & \operatorname{Re}\left(\mathrm{B}_{\mathrm{y}}^{(\mathrm{n})} \mathrm{B}_{\mathrm{z}}^{(\mathrm{n}) *}\right) & \operatorname{Re}\left(\left|\mathrm{B}_{\mathrm{z}}^{(\mathrm{n})}\right|^{2}\right) \\
0 & -\operatorname{Im}\left(\mathrm{B}_{\mathrm{x}}^{(\mathrm{n})} \mathrm{B}_{\mathrm{y}}^{(\mathrm{n}) *}\right) & -\operatorname{Im}\left(\mathrm{B}_{\mathrm{x}}^{(\mathrm{n})} \mathrm{B}_{\mathrm{z}}^{(\mathrm{n}) *}\right) \\
\operatorname{Im}\left(\mathrm{B}_{\mathrm{x}}^{(\mathrm{n})} \mathrm{B}_{\mathrm{y}}^{(\mathrm{n}) *}\right) & 0 & -\operatorname{Im}\left(\mathrm{B}_{\mathrm{y}}^{(\mathrm{n})} \mathrm{B}_{\mathrm{z}}^{(\mathrm{n}) *}\right) \\
\operatorname{Im}\left(\mathrm{B}_{\mathrm{x}}^{(\mathrm{n})} \mathrm{B}_{\mathrm{z}}^{(\mathrm{n}) *}\right) & \operatorname{Im}\left(\mathrm{B}_{\mathrm{y}}^{(\mathrm{n})} \mathrm{B}_{\mathrm{z}}^{(\mathrm{n}) *}\right) & 0
\end{array}\right)
$$

where $N$ denotes the number of spectral matrices averaged over time. $B_{\mathrm{x}}^{(n)}, B_{\mathrm{y}}^{(n)}$ and $B_{\mathrm{z}}^{(n)}$ denote the three orthogonal components of the $n$-th magnetic field Fourier spectra in the MAG coordinate system for the obtained waveform by PWE/WFC.

$A$ can be decomposed by SVD as follows:

$$
A=U \cdot W \cdot V^{\mathrm{T}},
$$

where $U, W$, and $V^{\mathrm{T}}$ denote a $6 \times 3$ matrix, a $3 \times 3$ of three nonnegative singular values, and a $3 \times 3$ matrix with orthonormal rows, respectively. As reported by Santolík et al. (2003), the wave normal polar angle $\theta_{k}$, polarization ellipse $L_{\mathrm{p}}$ and planarity of polarization $F$ can be calculated as follows:

$$
\begin{aligned}
& \theta_{k}=\tan ^{-1}\left(\sqrt{v_{1}^{2}+v_{2}^{2}} / v_{3}^{2}\right), \\
& \left|L_{\mathrm{p}}\right|=w_{2} / w_{3}, \\
& F=1-\sqrt{w_{1} / w_{3}},
\end{aligned}
$$

where $w_{1}, w_{2}$, and $w_{3}$ denote the ascending series of singular values $W$, and $v_{1}, v_{2}$, and $v_{3}$ denote the corresponding order of the elements of matrix $V^{T} \cdot\left|L_{p}\right|=0$ and 1 indicate linear polarization and circular polarization, respectively. The sense of polarization can be determined from the sign of $a_{01}$, i.e., the positive and negative signs indicate that the wave is right-hand and left-hand polarized, respectively (Santolík et al. 2002). The planarity of polarization $F$ indicates the validity of the single planewave assumption. As demonstrated by Santolík et al. (2003), the SVD technique provides reasonable results when the single plane wave approximation is applicable. In that case, $F$ is near unity; otherwise, $F$ approaches zero.

It should be noted that $\theta_{k}$ has an ambiguity in the sign of polarity because the correlation between electric components parallel to the Poynting vector $S$ as follows:

$$
\left(\begin{array}{l}
S_{1} \\
S_{2} \\
S_{3}
\end{array}\right)=\left(\begin{array}{c}
\hat{E}_{\mathrm{y}} \hat{B}_{\mathrm{z}}^{*}-\hat{E}_{\mathrm{z}} \hat{B}_{\mathrm{y}}^{*} \\
-\hat{E}_{\mathrm{x}} \hat{B}_{\mathrm{z}}^{*}+\hat{E}_{\mathrm{z}} \hat{B}_{\mathrm{x}}^{*} \\
\hat{E}_{\mathrm{x}} \hat{B}_{\mathrm{y}}^{*}-\hat{E}_{\mathrm{y}} \hat{B}_{\mathrm{x}}^{*}
\end{array}\right),
$$

where $\hat{E}_{\mathrm{x}}, \hat{E}_{\mathrm{y}}$, and $\hat{E}_{\mathrm{z}}$ denote the three orthogonal components of the electric field Fourier spectra in the MAG coordinate system (Santolík et al. 2010). Because PWE/ WFC measures only two components of the electric field in the spin plane of Arase, we estimate the spin axis component $\hat{E}_{\mathrm{z}}$ under the assumption that $\boldsymbol{E} \cdot \boldsymbol{B}=0$. Here, $\boldsymbol{E}$ and $\boldsymbol{B}$ are the vectors of complex amplitudes of electric and magnetic fields.

We employ the LA_SVD procedure to perform SVD, which is implemented in the Linear Algebra PACKage (LAPACK) in IDL. Detailed descriptions of the LA_SVD procedure can be found on the website of L3 Harris Geospatial (https://www.l3harrisgeospatial.com/docs/la_svd. $\mathrm{html}$ ). If the singular value decomposition does not converge, ISEE_Wave automatically stops wave property calculations to prevent the user from obtaining a misleading result.

The data load routines are implemented separately from the core routines of the ISEE_Wave. Therefore, users can easily replace them with additional IDL procedure files (.pro) for waveform data from other satellites/instruments as well as ULF/ELF waveform data from EFD/MGF instruments aboard Arase. We will put a button on GUI to switch data load routines in a future update.

\section{Functions of ISEE_Wave}

When ISEE_Wave is launched, two panels appear. The OFA panel displays electric and magnetic field spectra observed by the PWE/OFA instrument aboard Arase (Fig. 2). OFA continuously measures electric and 
magnetic field auto-spectra below $20 \mathrm{kHz}$ in the nominal mode. The time resolution of wave power spectra observed by OFA is $1 \mathrm{~s}$ in the nominal mode; therefore, the observed spectra are useful for obtaining the entire VLF/ELF plasma wave activity (Matsuda et al. 2018). Furthermore, the history of waveform measurement by PWE/WFC is displayed in the OFA panel. Asterisks in the $65 k$ and WP panels in Fig. 2 present the timings when the chorus burst mode and SWPIA mode, respectively, were invoked onboard. Users can visualize the OFA spectra and waveform measurement history for user-specified time interval, which can be chosen using the top two text boxes and five buttons on the OFA panel.

The WFC panel provides GUI functions for advanced waveform analysis and displays the analysis results. When users press the Get Time Interval from OFA button (Fig. 3a), they can select a time-interval for waveform analysis by mouse gestures in the OFA panel. When the Start Calculation button is pressed, ISEE_Wave starts waveform analysis for data in a specified time period. After completing the waveform analysis, the results are displayed on the left side of the WFC panel. From top to bottom, the electric field power spectra, magnetic field power spectra, wave normal angle, polarization, planarity, and Poynting vector angle are displayed. Details of the GUI functions of the WFC panel are provided in Sections "Parameters of spectral matrix calculation" to "Output".

\section{Parameters of spectral matrix calculation}

The type of fast Fourier transform (FFT) window (Hanning or Hamming window), size of the FFT window, overlap length, and number of spectral matrices for averaging $\left(N_{\text {average }}\right)$ can be specified at the top of the WFC panel (Fig. 3b). Users should specify the appropriate analysis parameters according to the wave frequency range of the target and the purpose of analysis. Fig. 4 presents the definitions and the relationship between these parameters. If Stride and Window length are equal, the waveforms are separated by the length of Window length and transformed to Fourier spectra by the STFT without overlap (Fig. $4 \mathrm{a}$ ). When $N_{\text {average }}=3$, three consecutive spectral matrices are averaged to compute the ensemble-averaged spectral matrices. If Stride is set to half of the Window length, then the STFT is performed for time windows that overlap for half of them (Fig. 4b). The time resolution of the result is twice the result without overlapping. Note that the spectral matrices calculated by overlapping STFT are highly correlated with neighboring spectral matrices; the dynamic range of the planarity therefore appears to be pseudo-enhanced. The spectral matrix based on the original definition should be obtained from the STFT without overlapping.
Fig. 5 presents the results for different FFT window sizes: (a) 1024 points $(\delta f=64 \mathrm{~Hz})$ and (b) 4096 points $(\delta f=16 \mathrm{~Hz})$, where $\delta f$ denotes the frequency resolution of the calculated Fourier spectra. The overlap length and $N_{\text {average }}$ are the same, (i.e., 1024 points overlapping and three averages). As illustrated in the figure, the frequency resolution is improved in Fig. 5b, and the fine structures of whistler-mode chorus waves can be viewed more clearly.

Fig. 6 presents the results for different $N_{\text {average }}$ values, i.e., 3 and 10, presented in Figs. $6 \mathrm{a}$ and b, respectively. The FFT window size and the overlap length are the same, i.e., 4096 points and 1024 points, respectively. By averaging the spectral matrices, scatter of the resultant parameters is suppressed, and the results are smoothed. As displayed in the Planarity panel, the dynamic range of the results is improved. In other words, the signalto-noise ratio of the spectral matrices is improved by the averaging. Therefore, the significance of the wave normal angle and polarization analysis results are improved by increasing $N_{\text {average }}$. However, it is important to note that increasing $N_{\text {average }}$ reduces the time resolution.

\section{Support lines}

ISEE_wave can overlay support lines on plot panels to compare the wave frequency and electron cyclotron frequency. ISEE_Wave calculates local electron and ion cyclotron frequencies from DC magnetic field measurement data obtained by MGF aboard Arase (Matsuoka et al. 2018b). Users can enable or disable the drawing of each support line by editing the Support lines table displayed in Fig. 3c. Moreover, the line styles and colors can be changed in the same way. The selected support lines are overlayed on the wave property analysis results. The local electron cyclotron frequency $\left(f_{\mathrm{c}}\right)$, two related frequencies $\left(0.5 f_{\mathrm{c}}\right.$ and $\left.0.1 f_{\mathrm{c}}\right)$, and three ion cyclotron frequencies $\left(f c_{\mathrm{H}}, f c_{\mathrm{He}}\right.$ and $\left.f c_{\mathrm{O}}\right)$ are defined by default, where $f c_{\mathrm{H}}, f c_{\mathrm{He}}$ and $f c_{\mathrm{O}}$ denote the local proton cyclotron frequency, local helium ion cyclotron frequency, and local oxygen ion cyclotron frequency, respectively. Users can define new support lines and delete the existing support line definitions by clicking the Add New Line and Delete Selected Lines buttons, respectively.

\section{Orbital information}

Users can add text labels for the magnetic local time (MLT), magnetic latitude (MLAT), altitude, and McIlwain- $L$ value of Arase. By placing a check mark in each box presented in Fig. 3d, the corresponding orbital information is added below the plot. This orbital information 


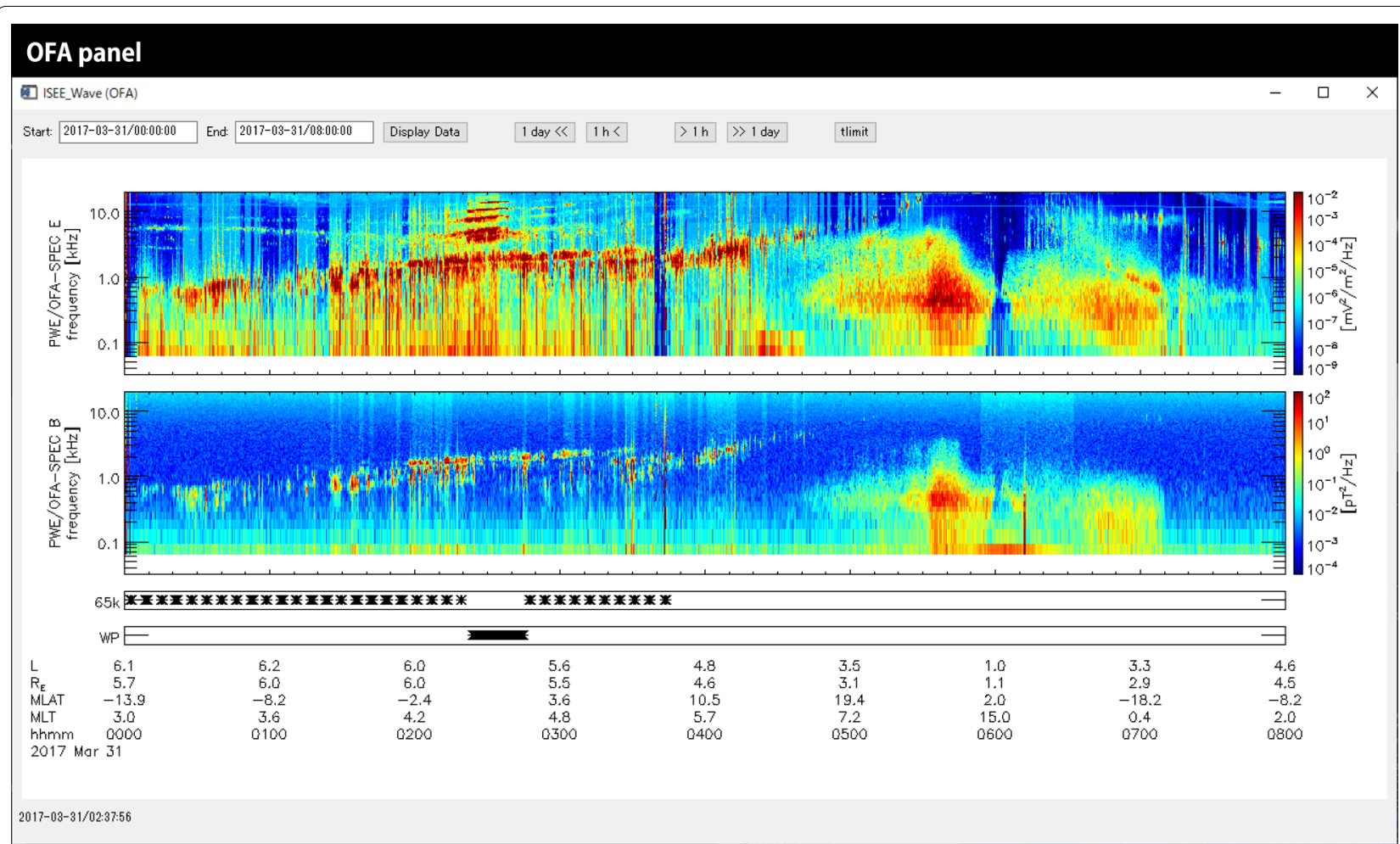

Fig. 2 A snapshot of the OFA pane/ when the time interval from 00:00:00 to 08:00:00 on 31 March 2017 is specified

is obtained from the Level-2 orbit data provided by the ERG Science Center (Miyoshi et al. 2018b). It should be noted that the MLT, MLAT and McIlwain- $L$ value provided from the Level-2 orbit data are calculated using the thirteenth generation of International Geomagnetic Reference Field (IGRF-13) model (Alken et al. 2021).

\section{$\mathrm{Y}$ - and Z-axes settings}

The Y- and Z-axes labels and their ranges can be changed by editing fields and checkboxes presented in Fig. 3e. By unchecking the Mask checkboxes, the original plots will be displayed instead of the masked results (for more details, see Section "Mask"). By unchecking the Plot checkboxes, the corresponding plots will not be displayed.

\section{Mask}

The wave analysis results can be masked by setting one or more thresholds for wave properties values. Users can set threshold values in the Mask tab of the WFC panel (Fig. 3f). Fig. 7 presents the results for three different thresholds of the magnetic field wave spectral intensity. As illustrated in Fig. $7 \mathrm{a}$ (data less than $10^{-3} \mathrm{pT}^{2} / \mathrm{Hz}$ are masked), $7 \mathrm{~b}$ (data less than $10^{-1} \mathrm{pT}^{2} / \mathrm{Hz}$ are masked), and $7 \mathrm{c}$ (data less than $10^{0} \mathrm{pT}^{2} / \mathrm{Hz}$ are masked), users can mask the noise floor of the instrument and weak wave events. This function is useful for extracting target wave events from the original wave spectra.

\section{Output}

The wave analysis results can be saved as image files, such as encapsuled post script (EPS) files and/or portable network graphics (PNG) files, and a tplot save file (Fig. 3g). By saving the results as a tplot save file, users can restore the digital values of the results using the tplot_restore procedure implemented in IDL/SPEDAS and thereby reuse the restored results for further analysis. This function is also useful for sharing results with other users.

\section{Scientific applications}

Figure $8 \mathrm{a}$ presents an example of waveform analysis using ISEE_Wave. We analyzed waveform data observed by PWE/WFC from 02:24:40 UT to 02:24:45 UT on March 31, 2017. We used a 2048 point Hanning window with 1024 points overlapping. We averaged three spectral matrices in time $\left(N_{\text {average }}=3\right)$. These parameters were set from the GUI of ISEE_Wave (see, Sect. "Parameters of spectral matrix calculation"). Arase was observing the geomagnetic equatorial region around dawn (MLAT $=0.0^{\circ}$ and MLT $=4.4 \mathrm{~h}$ ). The McIlwain $-L$ 


\section{WFC panel}

TI ISEE Wave (WFO

\begin{tabular}{|c|c|}
\hline \multicolumn{2}{|c|}{ Get Time Interval from OFA } \\
\hline Start: $2017-03-31 / 02: 24: 40$ & End: $2017-03-31 / 02: 24: 45$ \\
\hline
\end{tabular}
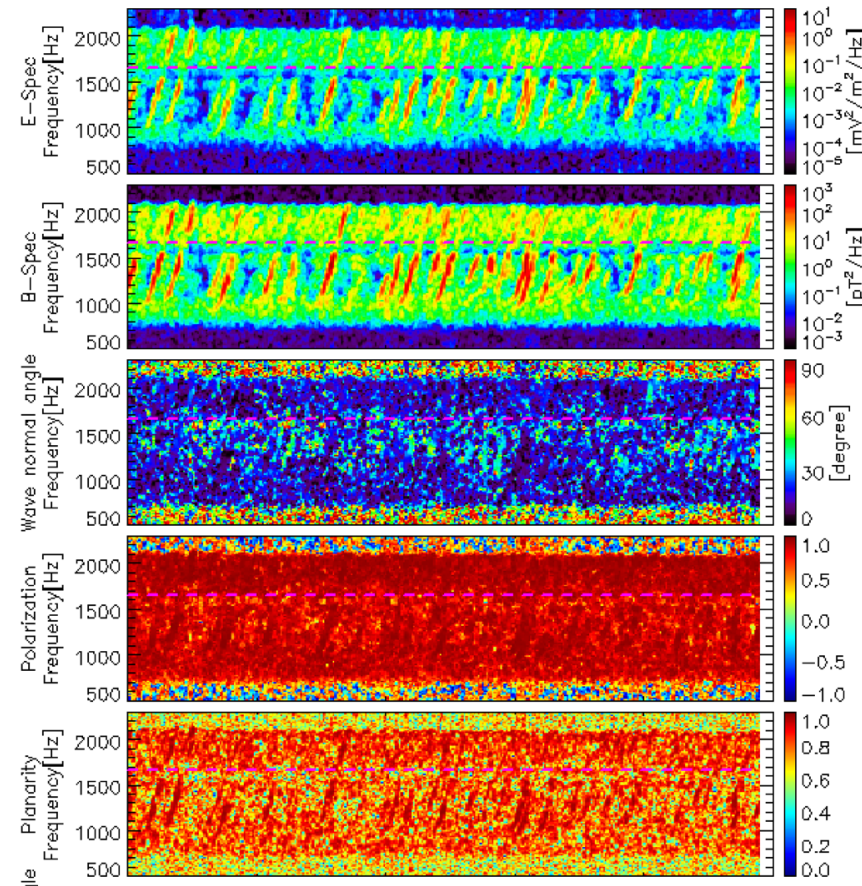

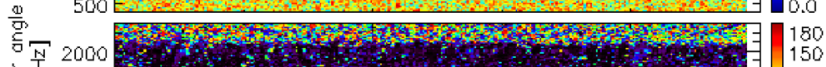

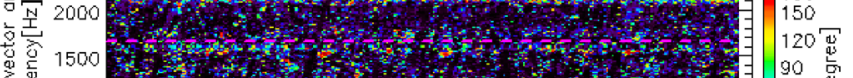

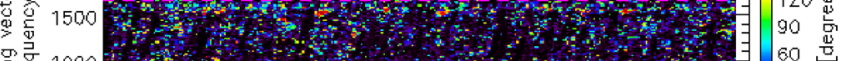

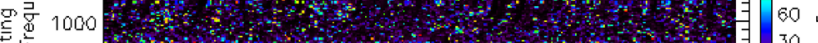

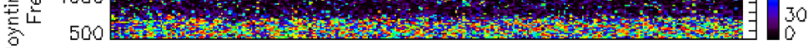

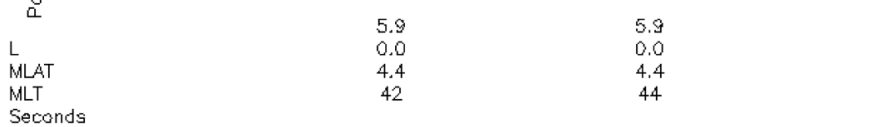

Seconds

2017 Mor 310224

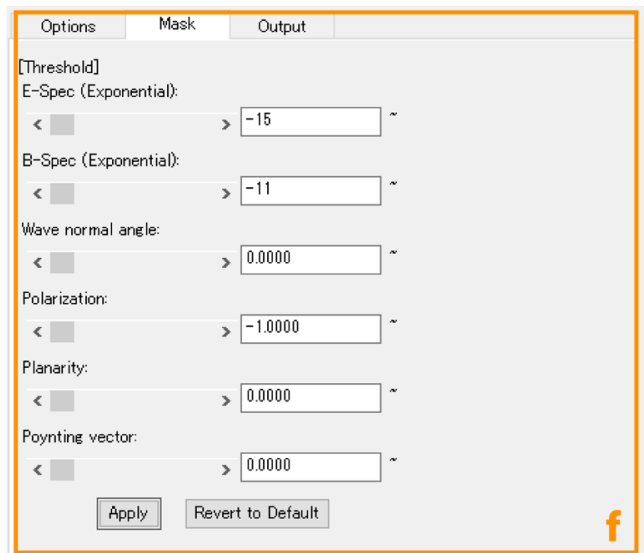

Fig. 3 A snapshot of the WFC pane/ when the time interval from 02:24:40 to 02:24:45 on 31 March 2017 is specified

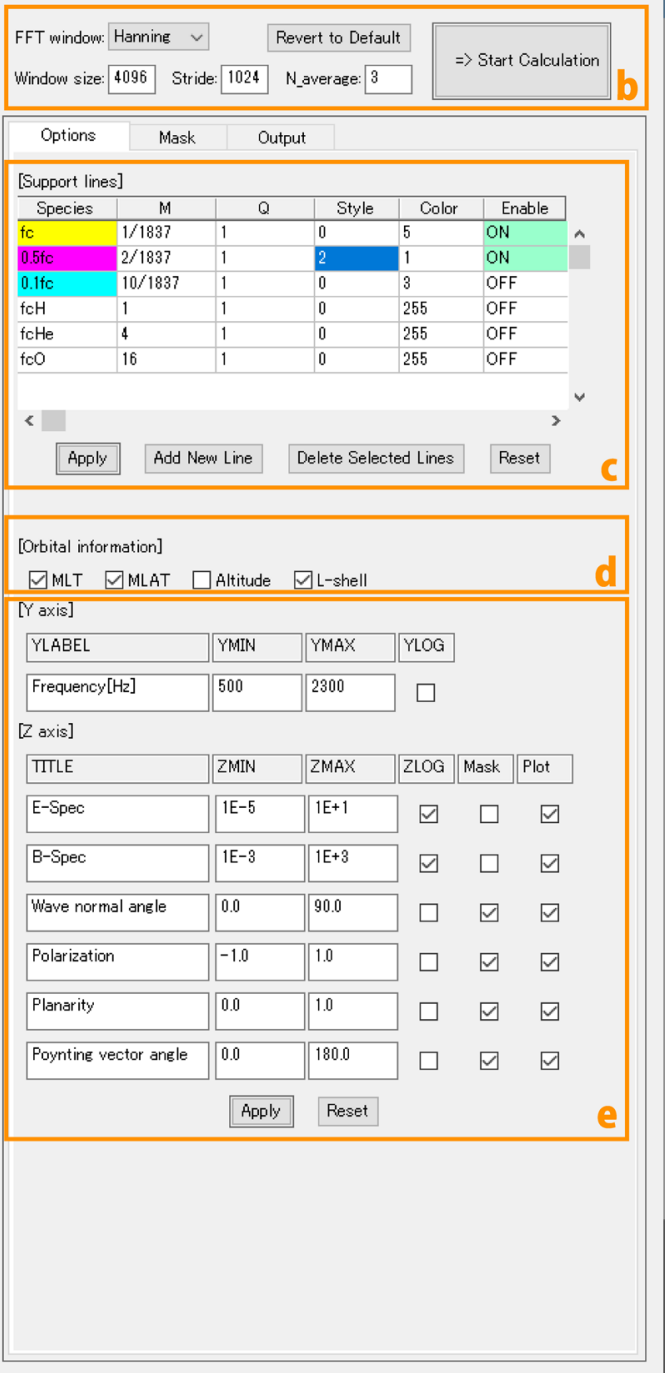

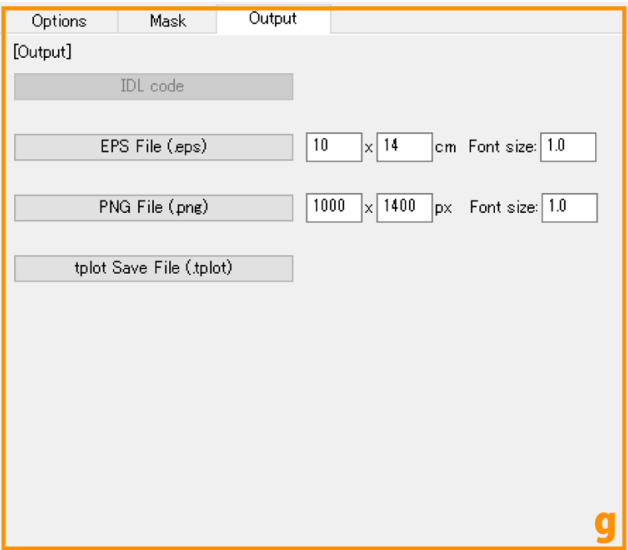




\section{a Stride $=$ Window length}

Waveform

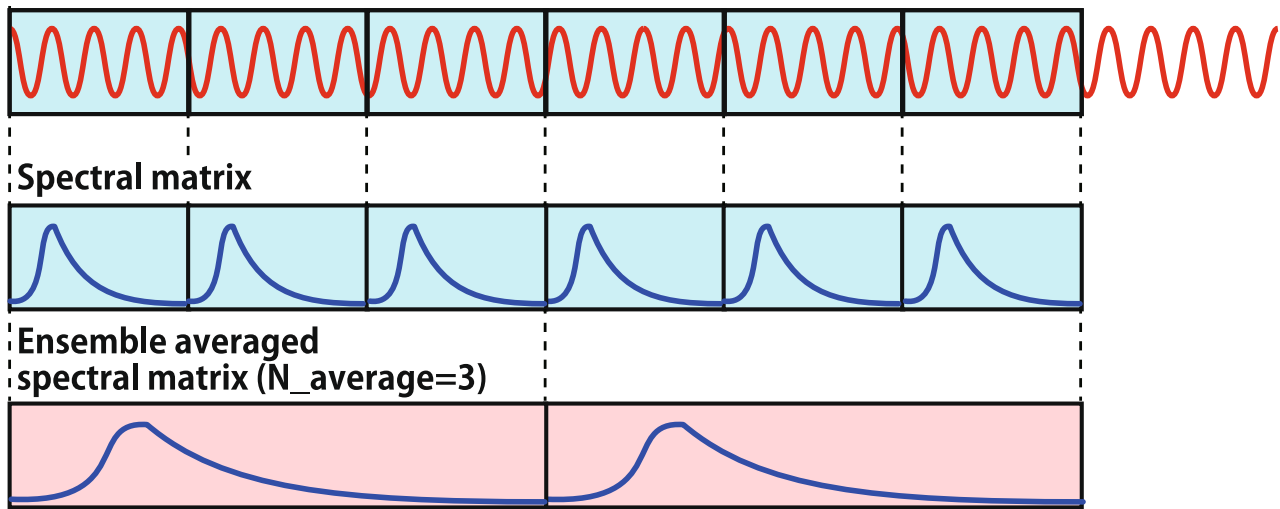

time

\section{b Stride $=$ Window length $\times 0.5$}

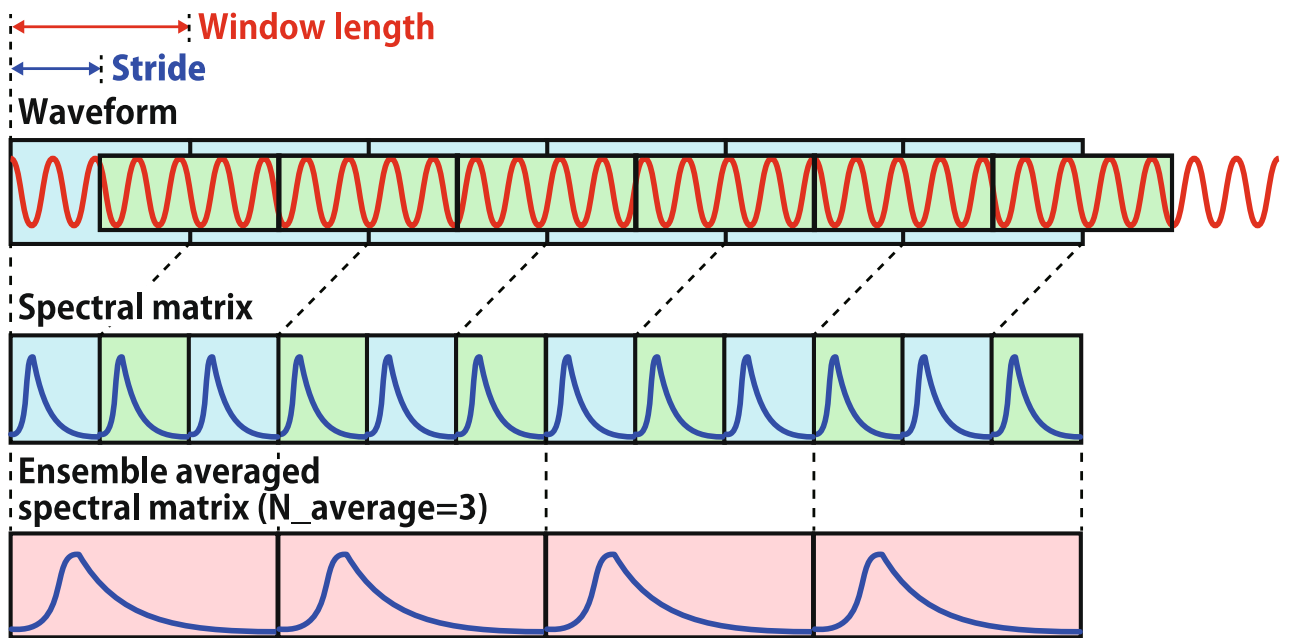

\section{$\stackrel{\text { time }}{\longrightarrow}$}

Fig. 4 The definitions and the relationship between the parameters of the short-time Fourier transform (window length, stride, and $N_{\text {average})}$ for (a) stride $=$ window length and $(\mathbf{b})$ stride $=$ window length $\times 0.5$

calculated with the IGRF-13 model was 5.9. These orbital parameters are displayed at the bottom of Fig. 8a (see, Sect. "Orbital information"). The magenta dashed lines represent half of the local electron cyclotron frequencies $\left(0.5 f_{\mathrm{c}}\right)$ calculated from the DC magnetic field measurement by MGF aboard Arase. These support lines were plotted by the function of ISEE_Wave (see, Sect. "Support lines"). As illustrated in the figure, discrete rising tone elements are clearly observed in this period. There is a gap around $0.5 f_{\mathrm{c}}$, suggesting that these are the typical lower-band and upper-band whistler-mode chorus waves in the terrestrial inner magnetosphere.

The Wave normal angle panel in Fig. 8a indicates that the wave normal angles of these whistler-mode chorus elements were oblique $\left(20-30^{\circ}\right)$ to the geomagnetic field line, while the Polarization panel indicates that the waves were almost purely right-hand polarized. The Planarity panel indicates that the planarity of these waves was 

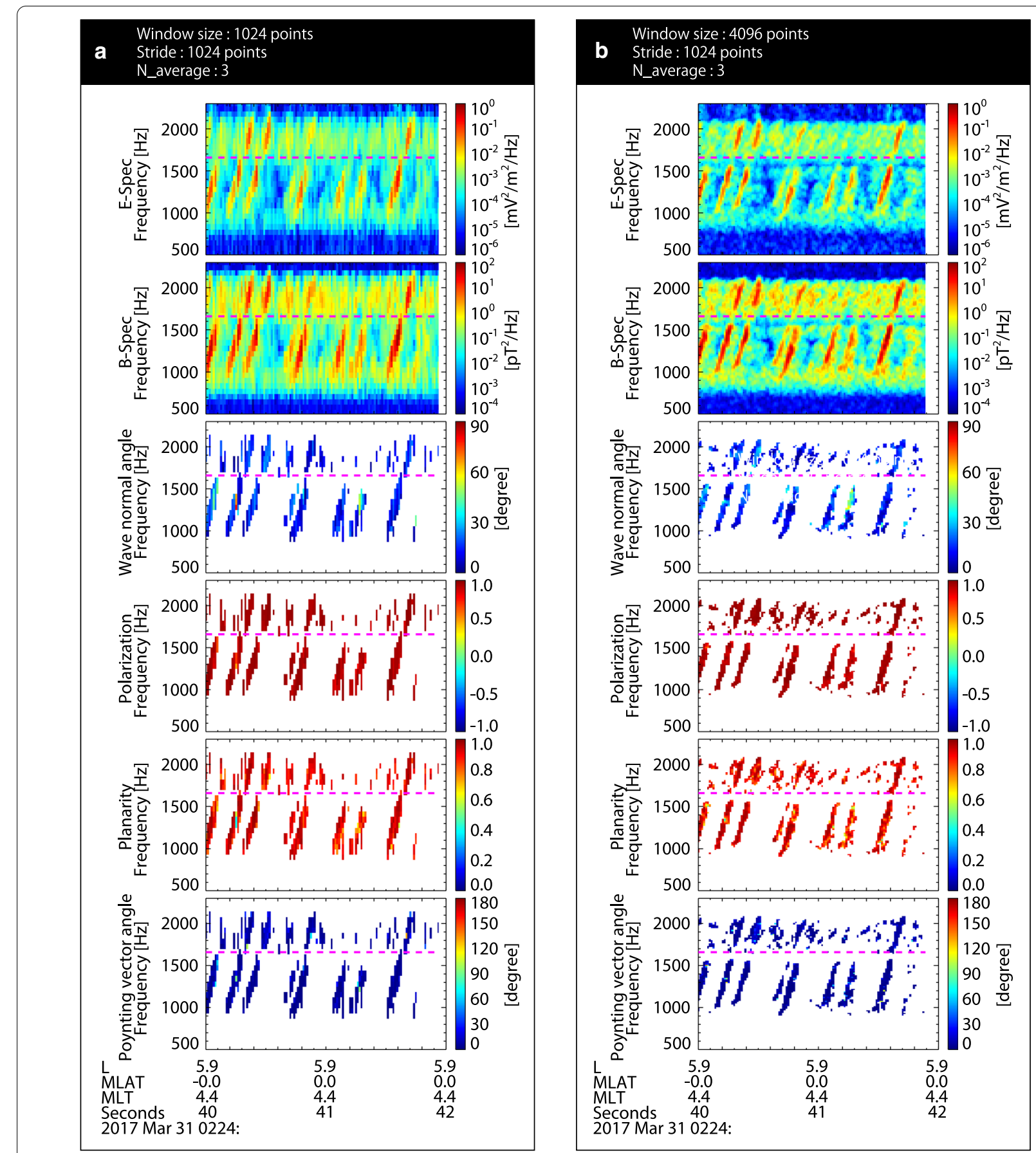

Fig. 5 Examples of results for different FFT window sizes; a 1024 points, b 4096 points

approximately 0.9 ; therefore, the approximation of the single plane wave approximation was satisfied for this event. The last panel Poynting vector angle indicates that the Poynting vector angles of these waves were almost $0^{\circ}$, suggesting northward propagation. For the last four panels in Fig. 8a, we applied masking under the condition of a magnetic field intensity of less than $10^{-1} \mathrm{pT}^{2} / \mathrm{Hz}$. This masking was also performed using the function of ISEE_ Wave (see, Sect. "Mask").

The source region of a typical whistler-mode chorus wave is thought to be at the geomagnetic equator (Nagano et al. 1996; LeDocq et al. 1998; Taubenschuss et al. 2016). Therefore, both northward and southward propagating waves should typically be observed around 

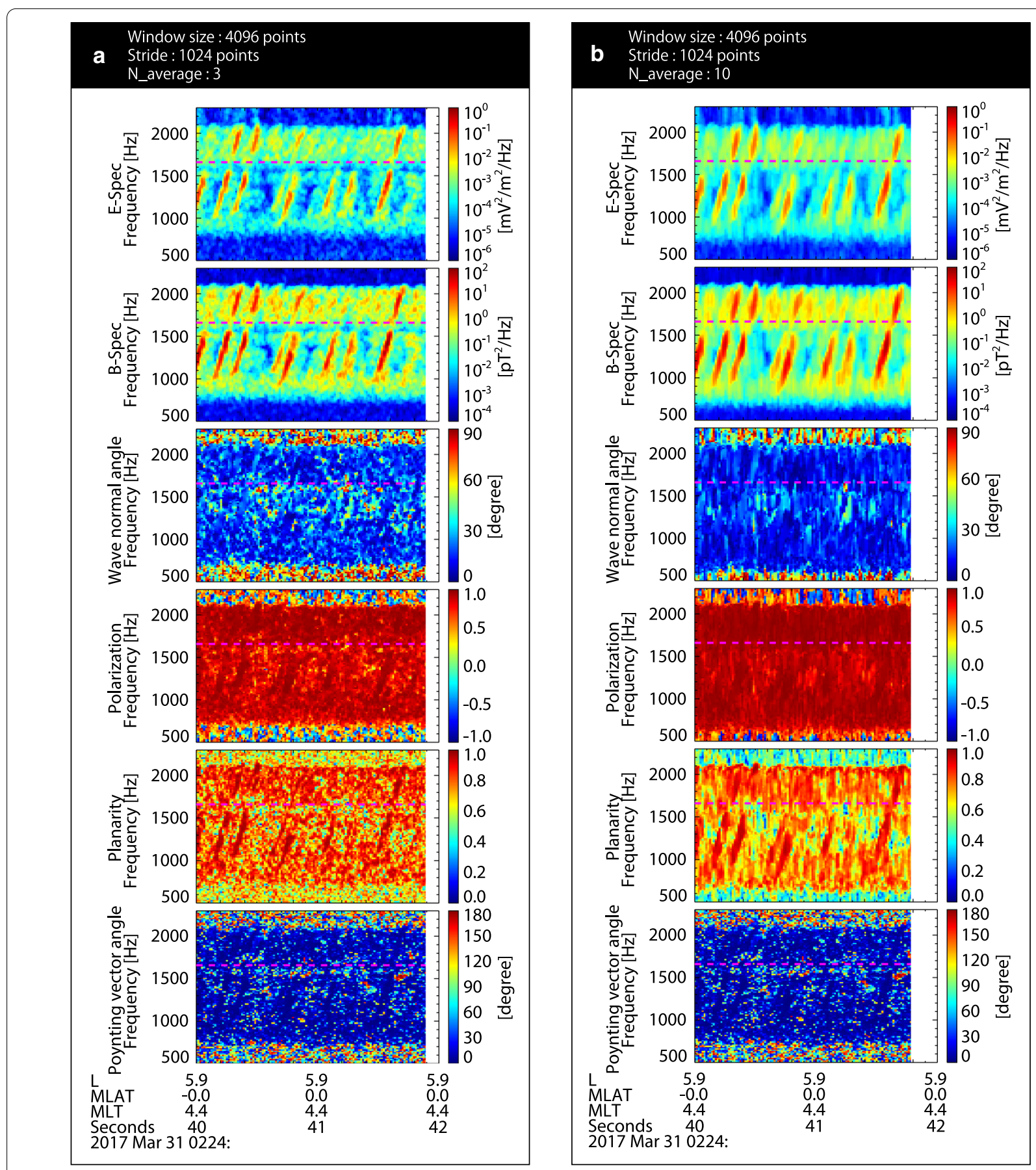

Fig. 6 Examples of results for different $N_{\text {average; }}$ a $N_{\text {average }}=3, \mathbf{b} N_{\text {average }}=10$

the geomagnetic equatorial region. However, all the whistler-mode chorus waves observed during the period propagated northward despite that these waves were observed at the geomagnetic equator $\left(\mathrm{MLAT}=0.0^{\circ}\right.$ ). The analysis result suggests that the source region of the observed whistler-mode chorus waves was located slightly south from the geomagnetic equator.
Figure 8b presents another example of waveform analysis using ISEE_Wave. We analyzed waveform data observed by PWE/WFC from 14:28:45 UT to 14:28:48 UT on July 18, 2018. We used a 2048 points Hanning window with 512 points overlapping. We averaged three spectral matrices in time $\left(N_{\text {average }}=3\right)$. Arase was observing the off-equatorial region around dusk 

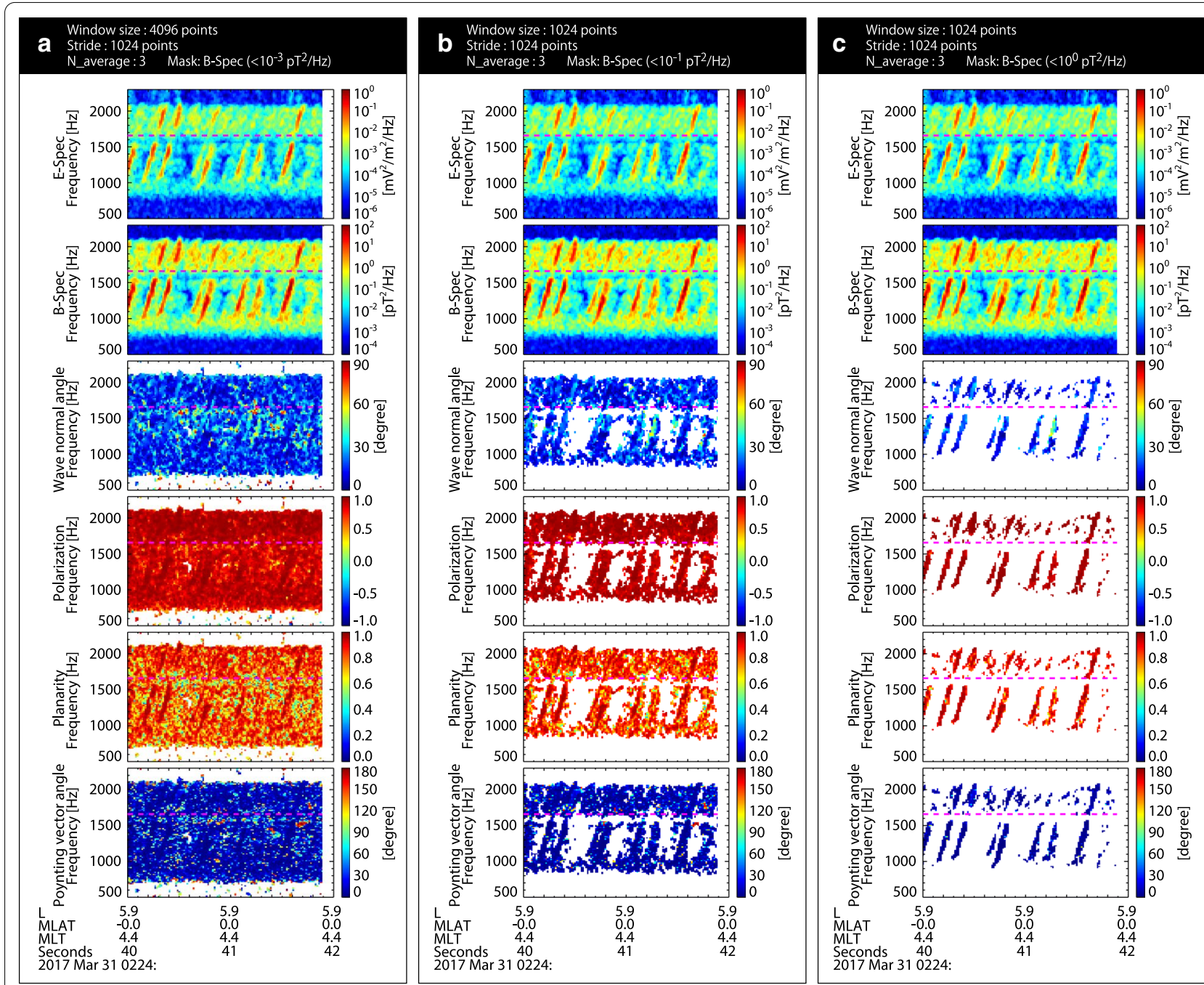

Fig. 7 Examples of results for three different masking thresholds of magnetic field spectral intensity. a Masked where the magnetic field spectral intensity was less than $10^{-3} \mathrm{pT}^{2} / \mathrm{Hz}$, $\mathbf{b}$ masked where magnetic field spectral intensity was less than $10^{-1} \mathrm{pT}^{2} / \mathrm{Hz}$ and $\mathbf{c}$ masked where magnetic field spectral intensity was less than $10^{0} \mathrm{pT}^{2} / \mathrm{Hz}$

$\left(\mathrm{MLAT}=7.3^{\circ}\right.$ and $\left.\mathrm{MLT}=18.7 \mathrm{~h}\right)$. The McIlwain $-L$ calculated with the IGRF-13 model was 1.2, suggesting that Arase was in the deep plasmasphere. For the last four panels in Fig. 8b, we applied masking under the condition of a magnetic field intensity of less than $10^{-2} \mathrm{pT}^{2} / \mathrm{Hz}$.

As illustrated in Fig. 8b, clear lightning whistler waves were observed in this period. From the last four panels in Fig. 8b, we observed the following wave properties:

- the waves were propagating with large wave angle (approximately $45^{\circ}$ ),

- the polarization of the waves was almost purely righthanded,
- the planarity of the waves was over 0.9; the single plane wave assumption well held for this event,

- the Poynting vector angles were almost $180^{\circ}$; therefore, the waves were propagating from high latitude region to the equator.

Lightning whistler waves are whistler-mode (right-hand polarized) waves excited by lightning discharges between the top and middle atmosphere, and propagate along geomagnetic field lines. The wave properties obtained by ISEE_Wave were consistent with the typical properties of lightning whistlers observed in the terrestrial plasmasphere. 

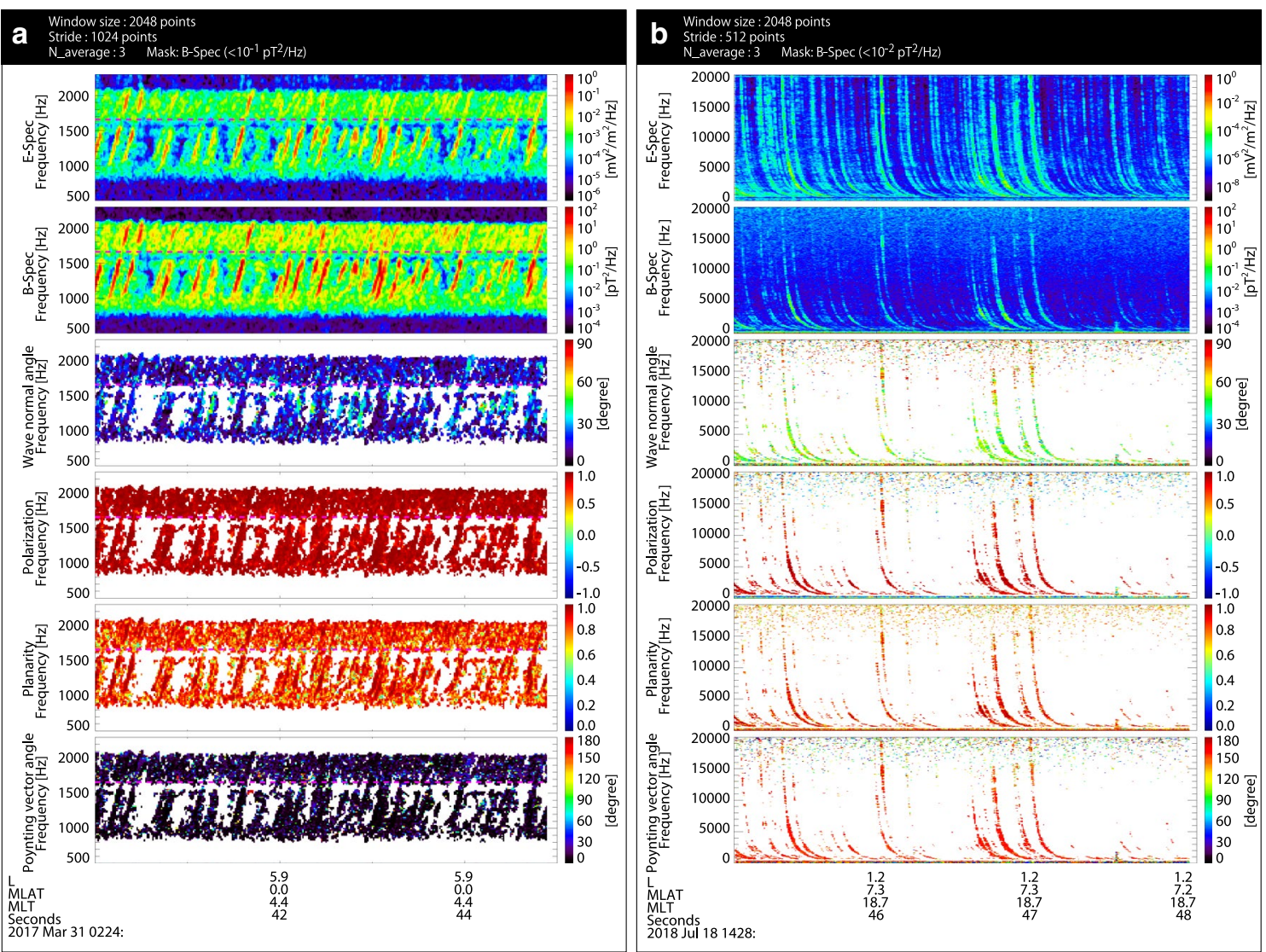

Fig. 8 Two examples of the results of wave property analysis by ISEE_Wave; a whistler-mode chorus waves, $\mathbf{b}$ lightning whistler waves

The computation time for analyzing $5 \mathrm{~s}$ waveform data of the event shown in Fig. 8a and $3 \mathrm{~s}$ waveform data of the event shown in Fig. 8b were approximately $15 \mathrm{~s}$ and $17 \mathrm{~s}$, respectively, using IDL 8.6.1 installed on a computer (Intel Core i9-10920X processor, 64 GB memory, and 64-bit Microsoft Windows 10 operating system). Time for data downloading from the data archive repository is not included in this computation time. It should be noted that we used a 2048 points Hanning window with 512 points overlapping for analyzing the event shown in Fig. 8b, while we used a 2048 points Hanning window with 1024 points overlapping for analyzing the event shown in Fig. 8a. Therefore, it takes longer time for analyzing the event shown in Fig. 8b although the duration of specified time interval is shorter than the event shown in Fig. 8a.

\section{Summary}

In this study, we have developed an interactive plasma wave analysis tool (ISEE_Wave) for visualizing advanced wave properties, such as electric and magnetic field wave power spectra, wave normal angles, polarization ellipses, planarity and Poynting vector angles. ISEE_Wave has been implemented as a part of the ERG plug-in for the IDL/
SPEDAS tool, and users can visualize these wave properties using GUIs in the IDL without using complicated commands. The internal processing of wave analysis is based on the SVD technique developed by Santolík et al. (2003). Derivation of spectral matrices and the transformation to MAG coordinates are implemented in ISEE_Wave. Several parameters for the STFT are extensively adjustable through the GUI of ISEE_Wave to allow users to perform appropriate frequency analysis on the target wave phenomena. Several examples were provided to demonstrate that we successfully visualized the wave properties of whistlermode chorus waves and those of lightning whistler waves observed by PWE/WFC with high time and frequency resolutions. ISEE_Wave also provides functions for overlaying support lines and manipulating various plot properties. The resultant plots can also be partially masked using a function of ISEE_Wave to clarify wave properties that the user focuses on. Users can export the wave property analysis result to image files and a tplot save file. The exported tplot save file allows users to restore the results in a commandline session of IDL/SPEDAS, which can then be used as an input for further analysis. ISEE_Wave can also be applied to waveform data obtained by other satellites, such as Van 


\section{Allen probes, MMS, and THEMIS, by replacing the data load routines.}

\begin{abstract}
Abbreviations
CUI: Character User Interface; DSI: Despun Sun sector Inertia; ELF: Extremely Low Frequency; EMFISIS: Electric and Magnetic Field Instrument Suite and Integrated Science; EMIC: Electromagnetic lon Cyclotron; ERG: Exploration of energization and Radiation in Geospace; FFT: Fast Fourier Transform; GUI Graphical User Interface; IDL: Interactive Data Language; IGRF: International Geomagnetic Reference Field; iPECMAN: Interface for a sPECtral Matrix ANalysis; ISEE_Wave: Institute for Space-Earth Environmental Research, Nagoya University - Plasma Wave Analysis Tool; MGF: Magnetic Field Experiment; MLAT: Magnetic Latitude; MLT: Magnetic Local Time; MMS: Magnetospheric Multiscale; OFA: Onboard Frequency Analyzer; PNG: Portable Network Graphics; PWE: Plasma Wave Experiment; PWI: Plasma Wave Investigation; QMF: Quadrature Mirror Filter; RPWS: Radio and Plasma Wave Science; SGA: Spinning satellite Geometry Axis; SPEDAS: Space Physics Environment Data Analysis System; STAFF-SA: Spatio-Temporal Analysis of Field Fluctuations, Spectrum Analyser; STFT: Short-time Fourier Transform; SVD: Singular Value Decomposition; SWPIA: Software-type Wave-particle Interaction Analyzer; THEMIS: Time History of Events and Macroscale Interactions during Substorms; VLF: Very Low Frequency; WFC: Waveform Capture.
\end{abstract}

\section{Acknowledgements}

We thank F. Ogushi and M. Okawa for their helpful support on the development of the ISEE_Wave. We are grateful to N. Umemura and T. Segawa for their technical help on the release of ISEE_Wave. Part of this work was done at the ERG-Science Center operated by ISAS/JAXA and ISEE/Nagoya University. The SPEDAS software (Angelopoulos et al. 2019) was used for the data analysis in this study.

\section{Authors' contributions}

SM led the development of ISEE_Wave. YM, SN, MK, and MS proposed the idea of functions implemented on ISEE_Wave. TH, Sl, and CJ cooperated operational test of ISEE_Wave and provided suggestions for improving usability of ISEE_Wave. SK collaborated with SM in the development of the core part of the plasma wave property calculation. YK was the principal investigator of PWE aboard Arase and provided waveform data obtained by WFC. AM was the principal investigator of MGF aboard Arase and provided DC magnetic field data. IS was a project manager of the ERG project. All authors read and approved the final manuscript.

\section{Funding}

This study was supported by Grants-in-Aid for Scientific Research (14J02108, 20K14546 and 20H01959) of Japan Society for the Promotion of Science (JSPS). This study was supported by JSPS Bilateral Open Partnership Joint Research Projects (JPJSBP120192504).

\section{Availability of data and materials}

Science data of the ERG (Arase) satellite were obtained from the ERG Science Center operated by ISAS/JAXA and ISEE/Nagoya University (Miyoshi et al. 2018c) (https://ergsc.isee.nagoya-u.ac.jp/index.shtml.en). The Arase satellite data are publicly available in the ERG Science Center. In the present study, we used Level-2 PWE/OFA v02.01 power spectrum data (Kasahara et al. 2018b), Level-2 PWE/WFC v00.02 waveform data (Kasahara et al. 2020a, b), Level-2 MGF v03.04 8 s data (Matsuoka et al. 2018b), Level-2 definitive orbit v2 data (Miyoshi et al. 2018b) and Level-2 spacecraft attitude data.

\section{Declarations}

Ethics approval and consent to participate

Not applicable.

\section{Competing interests}

The authors declare that they have no competing interests.

\section{Consent for publication}

Not applicable.

\section{Author details}

${ }^{1}$ Institute of Space and Astronautical Science, Yoshinodai, Sagamihara 252-5210, Japan. ${ }^{2}$ Institute for Space-Earth Environmental Laboratory, Nagoya University, Chikusa-ku, Nagoya 464-8601, Japan. ${ }^{3}$ Research Institute for Sustainable Humanosphere, Kyoto University, Gokasho, Uji 611-0011, Japan. ${ }^{4}$ Graduate School of Natural Science and Technology, Kanazawa University, Kakuma-machi, Kanazawa 920-1192, Japan. ${ }^{5}$ Data Analysis Center for Geomagnetism and Space Magnetism, Graduate School of Science, Kyoto University, Kitashirakawa-Oiwake Cho, Sakyo-ku, Kyoto 606-8502, Japan.

Received: 2 March 2021 Accepted: 30 April 2021

Published online: 20 May 2021

\section{References}

Alken P, Thébault E, Beggan CD, Amit H, Aubert J, Baerenzung J, Bondar TN, Brown WJ, Califf S, Chambodut A, Chulliat A, Cox GA, Finlay CC, Fournier A, Gillet N, Grayver A, Hammer MD, Holschneider M, Huder L, Hulot G, Jager T, Kloss C, Korte M, Kuang W, Kuvshinov A, Langlais B, Léger J-M, Lesur V, Livermore PW, Lowes FJ, Macmillan S, Magnes W, Mandea M, Marsal S, Matzka J, Metman MC, Minami T, Morschhauser A, Mound JE, Nair M, Nakano S, Olsen N, Pavón-Carrasco FJ, Petrov VG, Ropp G, Rother M, Sabaka TJ, Sanchez S, Saturnino D, Schnepf NR, Shen X, Stolle C, Tangborn A, Clausenffner-Clausen L, Toh H, Torta JM, Varner J, Vervelidou F, Vigneron P, Wardinski I, Wicht J, Woods A, Yang Y, Zeren Z, Zhou B (2021) International geomagnetic reference field: the thirteenth generation. Earth, Planets and Space 73(1):49. https://doi.org/10.1186/ s40623-020-01288-x

Angelopoulos V (2008) The THEMIS mission. Space Sci Rev 141(1):5. https://doi. org/10.1007/s11214-008-9336-1

Angelopoulos V, Cruce P, Drozdov A, Grimes EW, Hatzigeorgiu N, King DA, Larson D, Lewis JW, McTiernan JM, Roberts DA, Russell CL, Hori T, Kasahara Y, Kumamoto A, Matsuoka A, Miyashita Y, Miyoshi Y, Shinohara I, Teramoto M, Faden JB, Halford AJ, McCarthy M, Millan RM, Sample JG, Smith DM, Woodger LA, Masson A, Narock AA, Asamura K, Chang TF, Chiang C-Y, Kazama Y, Keika K, Matsuda S, Segawa T, Seki K, Shoji M, Tam SWY, Umemura N, Wang B-J, Wang S-Y, Redmon R, Rodriguez JV, Singer HJ, Vandegriff J, Abe S, Nose M, Shinbori A, Tanaka Y-M, UeNo S, Andersson L, Dunn P, Fowler C, Halekas JS, Hara T, Harada Y, Lee CO, Lillis R, Mitchell DL, Argall MR, Bromund K, Burch JL, Cohen IJ, Galloy M, Giles B, Jaynes AN, Le Contel O, Oka M, Phan TD, Walsh BM, Westlake J, Wilder FD, Bale SD, Livi R, Pulupa M, Whittlesey P, DeWolfe A, Harter B, Lucas E, Auster U, Bonnell JW, Cully CM, Donovan E, Ergun RE, Frey HU, Jackel B, Keiling A, Korth H, McFadden JP, Nishimura Y, Plaschke F, Robert P, Turner DL, Weygand JM, Candey RM, Johnson RC, Kovalick T, Liu MH, McGuire RE, Breneman A, Kersten K, Schroeder P (2019) The space physics environment data analysis system (SPEDAS). Space Sci Rev 215(1):9. https://doi.org/10.1007/ S11214-018-0576-4

Burch JL, Moore TE, Torbert RB, Giles BL (2016) Magnetospheric multiscale overview and science objectives. Space Sci Rev 199(1):5-21. https://doi. org/10.1007/s11214-015-0164-9

Colpitts C, Miyoshi Y, Kasahara Y, Delzanno GL, Wygant JR, Cattell CA, Breneman A, Kletzing C, Cunningham G, Hikishima M, Matsuda S, Katoh Y, Ripoll J-F, Shinohara I, Matsuoka A (2020) First direct observations of propagation of discrete chorus elements from the equatorial source to higher latitudes, using the Van Allen probes and Arase satellites. J Geophys Res Space Phys 125(10):2020-028315. https://doi.org/10.1029/ 2020JA028315

Cornilleau-Wehrlin N, Chauveau P, Louis S, Meyer A, Nappa JM, Perraut S, Rezeau L, Robert P, Roux A, De Villedary C, De Conchy Y, Friel L, Harvey CC, Hubert D, Lacombe C, Manning R, Wouters F, Lefeuvre F, Parrot M, Pinçon JL, Poirier B, Kofman W, Louarn P (1997) The Cluster spatio-temporal analysis of field fluctuations (STAFF) experiment. Space Sci Rev 79(1):107-136. https://doi.org/10.1023/A:1004979209565

Cornilleau-Wehrlin N, Chanteur G, Perraut S, Rezeau L, Robert P, Roux A, de Villedary C, Canu P, Maksimovic M, de Conchy Y, Hubert D, Lacombe C, Lefeuvre F, Parrot M, Pinçon JL, Décréau PME, Harvey CC, Louarn P, Santolik O, Alleyne HSC, Roth M, Chust T, Le Contel O, team S (2003) First results obtained by the Cluster STAFF experiment. Annales Geophysicae 21(2):437-456. https://doi.org/10.5194/angeo-21-437-2003 
Foster JC, Erickson PJ, Omura Y, Baker DN, Kletzing CA, Claudepierre SG (2017) Van allen probes observations of prompt mev radiation belt electron acceleration in nonlinear interactions with vlf chorus. J Geophys Res Space Phys 122(1):324-339. https://doi.org/10.1002/2016JA023429

Gurnett DA, Persoon AM, Randall RF, Odem DL, Remington SL, Averkamp TF, Debower MM, Hospodarsky GB, Huff RL, Kirchner DL, Mitchell MA, Pham BT, Phillips JR, Schintler WJ, Sheyko P, Tomash DR (1995) The Polar plasma wave instrument. Space Sci Rev 71(1):597-622. https://doi.org/10.1007/ BF00751343

Gurnett DA, Kurth WS, Kirchner DL, Hospodarsky GB, Averkamp TF, Zarka P, Lecacheux A, Manning R, Roux A, Canu P, Cornilleau-Wehrlin N, Galopeau P, Meyer A, Boström R, Gustafsson G, Wahlund J-E, Åhlen L, Rucker HO, Ladreiter HP, Macher W, Woolliscroft LC, Alleyne H, Kaiser ML, Desch MD, Farrell WM, Harvey CC, Louarn P, Kellogg PJ, Goetz K, Pedersen A (2004) In: Russell CT (ed) The Cassini Radio and Plasma Wave Investigation. Springer, Dordrecht, pp 395-463. https://doi.org/10.1007/ 978-1-4020-2774-1_6

Horne RB, Thorne RM, Glauert SA, Albert JM, Meredith NP, Anderson RR (2005) Timescale for radiation belt electron acceleration by whistler mode chorus waves. J Geophys Res Space Phys https://doi.org/10.1029/2004J A010811

Kasahara S, Miyoshi Y, Yokota S, Mitani T, Kasahara Y, Matsuda S, Kumamoto A, Matsuoka A, Kazama Y, Frey HU, Angelopoulos V, Kurita S, Keika K, Seki K, Shinohara I (2018) Pulsating aurora from electron scattering by chorus waves. Nature 554(7692):337-340. https://doi.org/10.1038/nature25505

Kasahara Y, Kasaba Y, Kojima H, Yagitani S, Ishisaka K, Kumamoto A, Tsuchiya F, Ozaki M, Matsuda S, Imachi T, Miyoshi Y, Hikishima M, Katoh Y, Ota M, Shoji M, Matsuoka A, Shinohara I (2018a) The plasma wave experiment (PWE) on board the Arase (ERG) satellite. Earth, Planets and Space 70(1):86. https://doi.org/10.1186/s40623-018-0842-4

Kasahara Y, Kojima H, Matsuda S, Ozaki M, Yagitani S, Shoji M, Nakamura S, Kitahara M, Miyoshi Y, Shinohara I (2018b) PWE/OFA instrument level-2 spectrum data of exploration of energization and radiation in geospace (ERG) Arase satellite, version v02.01, updated daily. ERG Science Center, Institute for Space-Earth Environmental Research, Nagoya University. https://doi.org/10.34515/DATA.ERG-08000. Accessed 2021-02-04

Kasahara Y, Kojima H, Matsuda S, Shoji M, Nakamura S, Kitahara M, Shinohara I, Miyoshi Y (2020a) The PWE/WFC instrument level-2 electric field waveform data of exploration of energization and radiation in geospace (ERG) Arase satellite, version v00.02, updated daily. ERG Science Center, Institute for Space-Earth Environmental Research, Nagoya University. https://doi. org/10.34515/DATA.ERG-09000. Accessed 2021-02-04

Kasahara Y, Kojima H, Matsuda S, Ozaki M, Yagitani S, Shoji M, Nakamura S, Kitahara M, Shinohara I, Miyoshi Y (2020b) The PWE/WFC instrument level-2 magnetic field waveform data of exploration of energization and radiation in geospace (ERG) Arase satellite, version v00.02, updated daily. ERG Science Center, Institute for Space-Earth Environmental Research, Nagoya University. https://doi.org/10.34515/DATA.ERG-09001. Accessed 2021-02-04

Kletzing CA, Kurth WS, Acuna M, MacDowall RJ, Torbert RB, Averkamp T, Bodet D, Bounds SR, Chutter M, Connerney J, Crawford D, Dolan JS, Dvorsky R, Hospodarsky GB, Howard J, Jordanova V, Johnson RA, Kirchner DL, Mokrzycki B, Needell G, Odom J, Mark D, Pfaff R, Phillips JR, Piker CW, Remington SL, Rowland D, Santolik O, Schnurr R, Sheppard D, Smith CW, Thorne RM, Tyler J (2013) The electric and magnetic field instrument suite and integrated science (EMFISIS) on RBSP. Space Sci Rev 179(1):127-181. https://doi.org/10.1007/s11214-013-9993-6

LeDocq MJ, Gurnett DA, Hospodarsky GB (1998) Chorus source locations from VLF Poynting flux measurements with the Polar spacecraft. Geophys Res Lett 25(21):4063-4066. https://doi.org/10.1029/1998GL900071

Martinez-Calderon C, Katoh Y, Manninen J, Kasahara Y, Matsuda S, Kumamoto A, Tsuchiya F, Matsuoka A, Shoji M, Teramoto M, Shinohara I, Shiokawa K, Miyoshi Y (2020) Conjugate observations of dayside and nightside VLF chorus and QP emissions between Arase (ERG) and Kannuslehto. Finland. J Geophys Res Space Phys 125(1):2019-026663. https://doi.org/10.1029/ 2019JA026663

Matsuda S, Kasahara Y, Kojima H, Kasaba Y, Yagitani S, Ozaki M, Imachi T, Ishisaka K, Kumamoto A, Tsuchiya F, Ota M, Kurita S, Miyoshi Y, Hikishima M, Matsuoka A, Shinohara I (2018) Onboard software of plasma wave experiment aboard Arase: instrument management and signal processing of waveform capture/onboard frequency analyzer. Earth, Planets and Space 70(1):75. https://doi.org/10.1186/s40623-018-0838-0

Matsuoka A, Teramoto M, Nomura R, Nosé M, Fujimoto A, Tanaka Y, Shinohara M, Nagatsuma T, Shiokawa K, Obana Y, Miyoshi Y, Mita M, Takashima T, Shinohara I (2018a) The Arase (ERG) magnetic field investigation. Earth, Planets and Space 70(1):43. https://doi.org/10.1186/s40623-018-0800-1

Matsuoka A, Teramoto M, Imajo S, Kurita S, Miyoshi Y, Shinohara I (2018b) The MGF instrument level-2 spinfit magnetic field data of exploration of energization and radiation in geospace (ERG) Arase satellite, version v03.04, updated daily. ERG Science Center, Institute for Space-Earth Environmental Research, Nagoya University. https://doi.org/10.34515/DATA. ERG-06001. Accessed 2021-02-04

Mauk BH, Fox NJ, Kanekal SG, Kessel RL, Sibeck DG, Ukhorskiy A (2013) Science objectives and rationale for the radiation belt storm probes mission. Space Sci Rev 179(1):3-27. https://doi.org/10.1007/s11214-012-9908-y

Miyoshi Y, Shinohara I, Takashima T, Asamura K, Higashio N, Mitani T, Kasahara S, Yokota S, Kazama Y, Wang S-Y, Tam SWY, Ho PTP, Kasahara Y, Kasaba Y, Yagitani S, Matsuoka A, Kojima H, Katoh Y, Shiokawa K, Seki K (2018a) Geospace exploration project ERG. Earth, Planets and Space 70(1):101. https://doi.org/10.1186/s40623-018-0862-0

Miyoshi Y, Shinohara I, Jun C-W (2018b) The level-2 orbit data of exploration of energization and radiation in geospace (ERG) Arase satellite, version v03, updated daily. ERG Science Center, Institute for Space-Earth Environmental Research, Nagoya University. https://doi.org/10.34515/DATA.ERG12000. Accessed 2021-02-04

Miyoshi Y, Hori T, Shoji M, Teramoto M, Chang TF, Segawa T, Umemura N, Matsuda S, Kurita S, Keika K, Miyashita Y, Seki K, Tanaka Y, Nishitani N, Kasahara S, Yokota S, Matsuoka A, Kasahara Y, Asamura K, Takashima T, Shinohara I (2018c) The ERG science center. Earth, Planets and Space 70(1):96. https:// doi.org/10.1186/s40623-018-0867-8

Nagano I, Yagitani S, Kojima H, Matsumoto H (1996) Analysis of wave normal and Poynting vectors of the chorus emissions observed by Geotail. J Geomagnetism Geoelectricity 48(3):299-307. https://doi.org/10.5636/jgg.48.299

Němec F, Santolík O, Gereová K, Macúsová E, de Conchy Y, Cornilleau-Wehrlin $N(2005)$ Initial results of a survey of equatorial noise emissions observed by the Cluster spacecraft. Planetary Space Sci 53(1):291-298. https://doi. org/10.1016/j.pss.2004.09.055

Ozaki M, Miyoshi Y, Shiokawa K, Hosokawa K, Oyama S-I, Kataoka R, Ebihara Y, Ogawa Y, Kasahara Y, Yagitani S, Kasaba Y, Kumamoto A, Tsuchiya F, Matsuda S, Katoh Y, Hikishima M, Kurita S, Otsuka Y, Moore RC, Tanaka Y, Nosé M, Nagatsuma T, Nishitani N, Kadokura A, Connors M, Inoue T, Matsuoka A, Shinohara I (2019) Visualization of rapid electron precipitation via chorus element wave-particle interactions. Nat Commun 10(1):257. https:// doi.org/10.1038/s41467-018-07996-z

Reeves GD, Spence HE, Henderson MG, Morley SK, Friedel RHW, Funsten HO, Baker DN, Kanekal SG, Blake JB, Fennell JF, Claudepierre SG, Thorne RM, Turner DL, Kletzing CA, Kurth WS, Larsen BA, Niehof JT (2013) Electron acceleration in the heart of the Van Allen radiation belts. Science 341 (6149):991-994. https://doi.org/10.1126/science.1237743

Santolík O, Parrot M, Lefeuvre F (2003) Singular value decomposition methods for wave propagation analysis. Radio Sci https://doi.org/10.1029/2000R 5002523

Santolík O, Parrot M, Storey LRO, Pickett JS, Gurnett DA (2001) Propagation analysis of plasmaspheric hiss using Polar PWI measurements. Geophys Res Lett 28(6):1127-1130. https://doi.org/10.1029/2000GL012239

Santolík O, Pickett JS, Gurnett DA, Storey LRO (2002) Magnetic component of narrowband ion cyclotron waves in the auroral zone. J Geophys Res Space Phys 107(A12):17-11714. https://doi.org/10.1029/2001JA000146

Santolík O, Pickett JS, Gurnett DA, Menietti JD, Tsurutani BT, Verkhoglyadova O (2010) Survey of Poynting flux of whistler mode chorus in the outer zone. J Geophys Res Space Phys 115(A7) https://doi.org/10.1029/2009J A014925

Taubenschuss U, Santolík O, Breuillard H, Li W, Le Contel O (2016) Poynting vector and wave vector directions of equatorial chorus. J Geophys Res Space Phys 121(12):11912-11928. https://doi.org/10.1002/2016JA023389

\section{Publisher's Note}

Springer Nature remains neutral with regard to jurisdictional claims in published maps and institutional affiliations. 